\title{
NOTES ON ALGEBRAIC FUNCTIONS
}

\author{
GUAN KE-YING and LEI JINZHI
}

Received 11 October 2001

\begin{abstract}
Consideration of the monodromy group of the hypergeometric equation $z$ (1$z) w^{\prime \prime}+[\gamma-(1+\alpha+\beta) z] w^{\prime}-\alpha \beta w=0$, in the case of $\alpha=1 / 6, \beta=5 / 6, \gamma=7 / 6$, shows that the global hypergeometric function solution $\mathbf{F}(1 / 6 ; 5 / 6 ; 7 / 6 ; z)$ is nonalgebraic although it has only algebraic singularities. Therefore, the proposition given in $[2,4]$ that a function is algebraic if it has only the algebraic singularities on the extended $z$-plane is not true. Through introduction of the concept of singular element criterion for deciding when a function is algebraic on the basis of properties of its singularities is given.
\end{abstract}

2000 Mathematics Subject Classification: 30F99, 34M99.

1. Introduction. The theory of algebraic functions is a classical branch of mathematics [6, 7]. Algebraic functions play a very important role in the study of the integrability theory of ordinary differential equations [14, 15]. By definition, a function $w=w(z)$ is algebraic if it satisfies a polynomial equation $P(z, w)=0$. In practice, one desires criteria for deciding whether or not a given function is algebraic recourse to the definition. One famous characterization is that an algebraic function has a compact Riemann surface, and conversely, a Riemann surface $S$ is compact if and only if there exists an irreducible polynomial $P$ in two variables such that $P(\operatorname{proj} \omega, \omega)=0$, for all $\omega \in S$, where proj $\omega$ is the projection of $\omega$ to the Riemann sphere [3, 6, 7].

Another characterization of the algebraic function is that they have only algebraic singular points. By [2, 4], a singular point $z_{0}$ of $w(z)$ is said to be algebraic if the function can be represented in a neighborhood of $z_{0}$ by a Puiseaux series of the form

$$
w(z)=\sum_{n=N}^{\infty} a_{n}\left(z-z_{0}\right)^{n / k},
$$

where $k(k>0)$ and $N$ are integers, and $a_{N} \neq 0$. When $N<0$, this point is called a critical pole.

In [4], one find the statement "A function is said to be of algebraic type in a region if it has only algebraic singularities in this region. Obviously, on the whole plane, an algebraic type function is an algebraic function." In [2], there is a similar statement "Conversely, we will prove that every function, which has only poles and algebraic ramification singular points, must be an algebraic 
function." This statement certainly would provide a simple criterion for recognizing algebraic function in terms of their singularities. Unfortunately, we find no proof for the assertion in [2] or [4].

On the other hand, in [10], it is proved that "Assume that the boundary of a region $G$ consists of finitely many points $z_{1}, z_{2}, \ldots, z_{m}, \infty$, that a function $f(z)$ is at most $n$-valued on $G$ and can be continued analytically along any continuous curve without limit, and that it can be represented by the expansion $\sum_{p=\mu}^{\infty} A_{p}\left(z-z_{j}\right)^{p / v}$ ( $\mu$ is an integer, and $v$ is a natural number) in a neighborhood of $z_{j}$ and be represented by the expansion $\sum_{p=\mu^{\prime}}^{\infty} A_{p}^{\prime} z^{-p / v}$ in a neighborhood of $\infty$, then $f(z)$ is an algebraic function." Similar statements appear in $[1,11]$. Although the condition "finiteness of the number of values of the function" is emphasized as "necessary," there is no explanation to show why the condition is really necessary. Moreover, it is not easy to find a nonalgebraic function with only algebraic singular points. Therefore, it does not help to ask whether the condition on "the finiteness of the number of values of the function" is necessary, or whether it can be replaced by a condition on the number and nature of singularities.

In Section 2, an explicit example of a nonalgebraic function with only algebraic singularities is given. Thus, the condition "the function has only a finite number of values over each point of sphere" is indeed independent of the condition "the function has only algebraic singularities," and so the above mentioned statements in [2, 4] are false.

This being the case, can we still recover a simple criterion like that in the statement given in $[2,4]$ and based only on the properties of the singularities of the function? In Section 3, using the concept of a singular element, we prove that a global analytic function is algebraic if and only if it has only isolated singularities, the number of its singular elements is finite, and every singular element is algebraic.

2. The function $F(1 / 6 ; 5 / 6 ; 7 / 6 ; z)$. In order to give the counterexample, we consider the following hypergeometric differential equation

$$
z(z-1) w^{\prime \prime}+[\gamma-(1+\alpha+\beta) z] w^{\prime}-\alpha \beta w=0,
$$

in the particular case of

$$
\alpha=\frac{1}{6}, \quad \beta=\frac{5}{6}, \quad \gamma=\frac{7}{6} .
$$

Singular points of any solution of this equation can appear only at $z=0, z=1$, or $z=\infty$.

The following facts are known in the classical theory of hypergeometric equation $[4,9]$ : 
(I) For any values of $\alpha, \beta$, and $\gamma$, (2.1) always has a local holomorphic solution $F(\alpha ; \beta ; \gamma ; z)$, called a hypergeometric function, which can be represented by a power series which converges in the disc, $\|z\|<1$.

The facts (II), (III), and (IV) are for the particular case (2.2).

(II) In a neighborhood of $z=0$, it has a pair of local solutions

$$
\begin{aligned}
w_{11}(z) & =F\left(\frac{1}{6} ; \frac{5}{6} ; \frac{7}{6} ; z\right) \\
w_{12}(z) & =z^{1-\gamma} F(\alpha-\gamma+1 ; \beta-\gamma+1 ; 2-\gamma ; z) \\
& =\frac{1}{z^{1 / 6}}
\end{aligned}
$$

where $w_{12}(z)$ is in fact an algebraic solution. $w_{11}(z)$ and $w_{12}(z)$ form a basic set of solutions in the disc $\|z\|<1$.

(III) In a neighborhood of $z=1$, the equation has the following pair of solutions

$$
\begin{aligned}
& w_{21}(z)=F\left(\frac{1}{6} ; \frac{5}{6} ; \frac{5}{6} ; 1-z\right)=\frac{1}{z^{1 / 6}}, \\
& w_{22}(z)=(1-z)^{1 / 6} F\left(\frac{1}{3} ; 1 ; \frac{7}{6} ; 1-z\right),
\end{aligned}
$$

where $w_{22}(z)$ has an algebraic singularity at $z=1$. In the disc $\|z-1\|<$ 1 , the functions $w_{21}(z)$ and $w_{21}(z)$ form a basic set of solutions.

(IV) In a neighborhood of $z=\infty$, the equation has the following pair of solutions

$$
\begin{aligned}
& w_{31}(z)=\left(\frac{1}{z}\right)^{1 / 6} F\left(\frac{1}{6} ; 0 ; \frac{1}{3} ; \frac{1}{z}\right)=\frac{1}{z^{1 / 6}}, \\
& w_{32}(z)=\left(\frac{1}{z}\right)^{5 / 6} F\left(\frac{2}{3} ; 1 ; \frac{5}{3} ; \frac{1}{z}\right)
\end{aligned}
$$

where $z=\infty$ is an algebraic singular point of both $w_{31}(z)$ and $w_{32}(z)$ which form a basic set of solutions in the region $\|z\|>1$.

In a neighborhood of $z=1 / 2$, the solutions, $w_{11}(z), w_{12}(z), w_{21}(z)$, and $w_{22}(z)$ are all holomorphic. By direct calculation, we may check that the local basic solution set $\left(w_{11}(z), w_{12}(z)\right)$ can be represented as a linear combination of $\left(w_{21}(z), w_{22}(z)\right)$ via the matrix equation

$$
\left(\begin{array}{l}
w_{11}(z) \\
w_{12}(z)
\end{array}\right)=\mathbf{P}\left(\begin{array}{l}
w_{21}(z) \\
w_{22}(z)
\end{array}\right),
$$

where

$$
\mathbf{P}=\left(\begin{array}{cc}
\frac{2^{2 / 3} \sqrt{\pi} \Gamma(7 / 6)}{\Gamma(2 / 3)} & -1 \\
1 & 0
\end{array}\right)
$$


Similarly, $\left(w_{21}(z), w_{22}(z)\right)$ can be represented by $\left(w_{31}(z), w_{32}(z)\right)$ via the matrix equation

$$
\left(\begin{array}{l}
w_{21}(z) \\
w_{22}(z)
\end{array}\right)=\mathbf{Q}\left(\begin{array}{l}
w_{31}(z) \\
w_{32}(z)
\end{array}\right),
$$

where $\mathbf{Q}=\left(q_{i j}\right)_{2 \times 2}$, and

$$
\begin{gathered}
q_{11}=1, \quad q_{12}=0, \\
q_{21}=(-1)^{1 / 6} 2^{-1 / 3} 3^{-5 / 6}\left\{F\left(\frac{1}{3} ; 1 ; \frac{7}{6} ;-\frac{1}{2}\right)\left[3+3^{1 / 6} F\left(\frac{5}{6} ; \frac{2}{3} ; \frac{5}{3} ; \frac{2}{3}\right)\right]\right. \\
\left.-\frac{9}{14} 3^{1 / 6} F\left(\frac{5}{6} ; \frac{2}{3} ; \frac{5}{3} ; \frac{2}{3}\right) F\left(\frac{4}{3} ; 2 ; \frac{13}{6} ;-\frac{1}{2}\right)\right\}, \\
q_{22}=\frac{9}{28}(-1)^{1 / 6} F\left(\frac{4}{3} ; 2 ; \frac{13}{6} ;-\frac{1}{2}\right)-\frac{1}{2}(-1)^{1 / 6} F\left(\frac{1}{3} ; 1 ; \frac{7}{6} ;-\frac{1}{2}\right) .
\end{gathered}
$$

By continuing $F(1 / 6 ; 5 / 6 ; 7 / 6 ; z)$ analytically, we obtain a global analytic function (The definition of global analytical function can be seen in [12]) F(1/6; $5 / 6 ; 7 / 6 ; z)$. It is easy to see the following proposition.

Proposition 2.1. Locally, every single-valued branch of the global analytical function $\mathrm{F}(1 / 6 ; 5 / 6 ; 7 / 6 ; z)$ can be represented as a linear combination of the local basic set of solutions listed in (2.3), (2.4), or (2.5). Therefore, $\mathbf{F}(1 / 6 ; 5 / 6 ; 7 / 6$; z) can be treated as a global particular solution of (2.1).

Proposition 2.2. Every singular point of each single-valued branch of the global analytical function $\mathbf{F}(1 / 6 ; 5 / 6 ; 7 / 6 ; z)$ is algebraic. Moreover, these singular points can occur only at $z=0, z=1$, or $z=\infty$.

By the declaration in $[2,4]$, the global function $\mathbf{F}(1 / 6 ; 5 / 6 ; 7 / 6 ; z)$ should be an algebraic function. We now prove that this is not the case.

We calculate the monodromy group of the given equation in case (2.2). By the definition of monodromy group, we know that the Fuchsian type equation (2.1) has two generators, $\mathbf{M}_{\mathbf{1}}$ and $\mathbf{M}_{2}$, which are represented by two $2 \times 2$ constant matrices such that from any starting point $z_{0}$, which is located in the intersection region of the discs $\|z\|<1$ and $\|z-1\|<1$, if we continue $w_{11}(z)$ and $w_{12}(z)$ analytically along a circle path around $z=0$ in the anticlockwise direction, then, $z$ returns to its initial value $z_{0}$, these two solutions will have new values represented through their original values and $\mathbf{M}_{\mathbf{1}}$ as follows:

$$
\left(\begin{array}{l}
\tilde{w}_{11}\left(z_{0}\right) \\
\tilde{w}_{12}\left(z_{0}\right)
\end{array}\right)=M_{1}\left(\begin{array}{c}
w_{11}\left(z_{0}\right) \\
w_{12}\left(z_{0}\right)
\end{array}\right)
$$

and if we continue $w_{11}(z)$ and $w_{12}(z)$ along a circular path around $z=1$ in the anticlockwise direction, then, when $z$ returns to its initial value $z_{0}$, these two solutions will have new values represented through their original values 
and $\mathbf{M}_{2}$ as follows:

$$
\left(\begin{array}{l}
\hat{w}_{11}\left(z_{0}\right) \\
\hat{w}_{12}\left(z_{0}\right)
\end{array}\right)=M_{2}\left(\begin{array}{l}
w_{11}\left(z_{0}\right) \\
w_{12}\left(z_{0}\right)
\end{array}\right)
$$

From (2.3), we may see that

$$
M_{1}=\left(\begin{array}{cc}
1 & 0 \\
0 & e^{-\pi i / 3}
\end{array}\right)
$$

In order to calculate $\mathbf{M}_{2}$, we point out that, by (2.4), if we continue $w_{21}(z)$ and $w_{22}(z)$ along a circular path around $z=1$ in the counterclockwise direction, when $z$ returns to its initial value $z_{0}$, these two solutions will have new values represented through their original values as follows:

$$
\left(\begin{array}{l}
\hat{w}_{21}\left(z_{0}\right) \\
\hat{w}_{22}\left(z_{0}\right)
\end{array}\right)=M_{2}^{*}\left(\begin{array}{l}
w_{21}\left(z_{0}\right) \\
w_{22}\left(z_{0}\right)
\end{array}\right)
$$

where

$$
M_{2}^{*}=\left(\begin{array}{cc}
1 & 0 \\
0 & e^{\pi i / 3}
\end{array}\right) .
$$

By relation (2.6) between $\left(w_{11}(z), w_{12}(z)\right)$ and $\left(w_{21}(z), w_{22}(z)\right)$, we see that

$$
M_{2}=P M_{2}^{*} P^{-1}=\left(\begin{array}{cc}
(-1)^{1 / 3} & \frac{-2^{2 / 3}\left[-1+(-1)^{1 / 3}\right] \sqrt{\pi} \Gamma(7 / 6)}{\Gamma(2 / 3)} \\
0 & 1
\end{array}\right) .
$$

It is easy to check that,

$$
\left(M_{1}\right)^{6}=\left(M_{2}\right)^{6}=\left(M_{1} M_{2}\right)^{6}=\left(\begin{array}{ll}
1 & 0 \\
0 & 1
\end{array}\right) .
$$

This means that $z=0, z=1$, and $z=\infty$ are all algebraic singular points for any given local solution of the equation.

However, if we let

$$
M_{3}=M_{1} M_{2}^{-1}=\left(\begin{array}{cc}
-(-1)^{2 / 3} & \frac{2^{2 / 3}\left[1+(-1)^{2 / 3}\right] \sqrt{\pi} \Gamma(7 / 6)}{\Gamma(2 / 3)} \\
0 & -(-1)^{2 / 3}
\end{array}\right),
$$

we have

$$
\left(M_{3}\right)^{6}=\left(\begin{array}{cc}
1 & \frac{62^{2 / 3}\left[-1+(-1)^{1 / 3}\right] \sqrt{\pi} \Gamma(7 / 6)}{\Gamma(2 / 3)} \\
0 & 1
\end{array}\right) .
$$




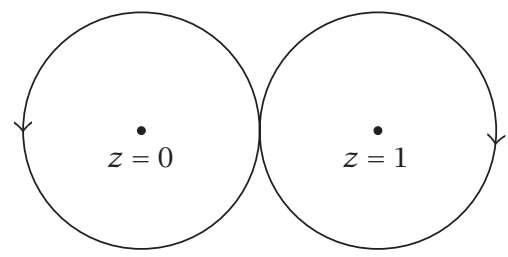

FIGURE 2.1. A directed path $L$.

Note that the monodromy matrix $M_{3}$ represents the operation of continuing a local solution along the directed and closed path $L$ formed by two tangent circles: one is around $z=1$ with clockwise direction, and the other is around $z=0$ with counterclockwise direction (see Figure 2.1).

If one continues the system $\left(w_{11}(z), w_{12}(z)\right)$ along $L$, then on returning to $z_{0}$ this system will have new values which are related to their original values as follows

$$
\left(\begin{array}{l}
\bar{w}_{11}\left(z_{0}\right) \\
\bar{w}_{12}\left(z_{0}\right)
\end{array}\right)=M_{3}\left(\begin{array}{l}
w_{11}\left(z_{0}\right) \\
w_{12}\left(z_{0}\right)
\end{array}\right)
$$

If we set

$$
\delta=\frac{62^{2 / 3}\left[-1+(-1)^{1 / 3}\right] \sqrt{\pi} \Gamma(7 / 6)}{\Gamma(2 / 3)}(\neq 0),
$$

then for any positive integer $n$,

$$
\left(M_{3}\right)^{6 n}=\left(\begin{array}{cc}
1 & n \delta \\
0 & 1
\end{array}\right)
$$

From (2.19) and (2.21) we see that, if we continue the local solution, $w_{11}(z)$, along the above mentioned path $L$ repeatedly $6 n$ times, then the new value $\check{w}_{11}\left(z_{0}\right)$ of $w_{11}(z)$ at $z_{0}$ is

$$
\breve{w}_{11}\left(z_{0}\right)=w_{11}\left(z_{0}\right)+n \delta w_{12}\left(z_{0}\right) \text {. }
$$

For instance, if we let $z_{0}=1 / 2$, we have

$$
\begin{aligned}
\check{w}_{11}\left(\frac{1}{2}\right) & =w_{11}\left(\frac{1}{2}\right)+n \delta w_{12}\left(\frac{1}{2}\right) \\
& =\frac{\sqrt{\pi} \Gamma(7 / 6)}{2^{1 / 6} \Gamma(2 / 3)}+2^{1 / 6} n \delta .
\end{aligned}
$$


Since the positive integer $n$ is arbitrarily given, we see that $\mathbf{F}(1 / 6 ; 5 / 6 ; 7 / 6 ; z)$ has infinitely many different values at $z_{0}$. Therefore, we obtained the following proposition.

Proposition 2.3. Since it assumes infinitely many values over $z_{0}=1 / 2$ the global hypergeometric function $\mathbf{F}(1 / 6 ; 5 / 6 ; 7 / 6 ; z)$ is not algebraic, although it has only algebraic singularities.

Note: there are in fact other ways to verify that $\mathbf{F}(1 / 6 ; 5 / 6 ; 7 / 6 ; z)$ is not algebraic, for instance, by the classification of hypergeometric equations with a full set of algebraic solutions based on Schwarz's differential invariant (see [5, 8, 9, 13]). (Before the referee's comment, we had not noticed this method though we had browsed the related part of [9].) It has been verified that $(1 / 6,5 / 6,7 / 6)$ does not correspond to an entry of Schwarz's list.

3. A decision criterion for algebraic function. Consider further the example of $F(1 / 6 ; 5 / 6 ; 7 / 6 ; z)$. From the traditional point of view, it seems that this infinitely many valued function has only three isolated singularities. However, at each singular point it has infinitely many different Puiseux series corresponding to different branches, in other words, as a whole, the function $\mathbf{F}(1 / 6 ; 5 / 6 ; 7 / 6 ; z)$ cannot be represented uniquely by a fixed Puiseux series in a neighborhood of a given singular point. In general, different branches of a multiplevalued function may show different singular properties at a given singular point, if this function has several singularities located at different points. In this case, it is imprecise to speak of the property of a given singular point without specifying the branch being considered. For example, it is not completely precise to say that the global function $\mathbf{F}(1 / 6 ; 5 / 6 ; 7 / 6 ; z)$ has only three algebraic singularities.

If we introduce the further concept of singular element in addition to that of analytic function element in the study of global analytical functions, as is done in $[11,16]$, then we may describe the singularity with greater precision.

For $z_{0} \in \hat{C}(\hat{C}=C \cup \infty)$, we say that the ordered pair $\left(f(z), z_{0}\right)$ is a singular element of a given global analytic function $\mathbf{F}(z)$ if $f(z)$ consists of branches of $\mathbf{F}(z)$ satisfying the following conditions:

(i) $z_{0}$ is the common isolated singular point of every branch of $f(z)$, in a small punctured disc $D_{0}=\left\{0<\left\|z-z_{0}\right\|<\epsilon\right\}$, any two analytic function elements of $f(z)$ can be continued to each other along a path in $D_{0}$;

(ii) no other analytic function element of $\mathbf{F}(z)$ can be obtained by continuation of an analytic function element of $f(z)$ along a path in $D_{0}$.

If in a neighborhood of $z_{0}, f(z)$ can be represented by a Puiseux series, that is,

$$
f(z)=\sum_{n=n_{0}}^{\infty} a_{n}\left(z-z_{0}\right)^{n / k}, \quad k \in N, a_{n_{0}} \neq 0,
$$


then the singular element $\left(f(z), z_{0}\right)$ is said to be algebraic. In this way, we may say more exactly that the global analytic function $F(1 / 6 ; 5 / 6 ; 7 / 6 ; z)$ has only three isolated singular points and infinitely many singular elements, and all the singular elements are algebraic.

Now, we may prove a theorem that gives a criterion for deciding whether or not a function is algebraic on the basis of its behavior at singularities.

THEOREM 3.1. A global analytic function $\mathbf{F}(z), z \in \hat{C}(\hat{C}=C \cup \infty)$ is algebraic if and only if, all its singular points are isolated, the number of singular elements is finite, and every singular element is algebraic.

Proof. It is obvious that when $\mathbf{F}(z), z \in \hat{C}(\hat{C}=C \cup \infty)$ is algebraic, then every singular point of it is isolated, the number of its singular elements is finite, and every singular element is algebraic. Hence we prove only the converse part of the theorem.

Assume that the global analytic function $\mathbf{F}(z), z \in \hat{C}(\hat{C}=C \cup \infty)$ has the following singular elements:

$$
\left(f_{1}(z), z_{1}\right),\left(f_{2}(z), z_{2}\right), \ldots,\left(f_{m}(z), z_{m}\right), \quad z_{j} \in \hat{C}
$$

where

$$
f_{j}(z)=\sum_{n=n_{j}}^{\infty} a_{j, n}\left(z-z_{j}\right)^{n / k_{j}}, \quad a_{j, n_{j}} \neq 0, j=1,2, \ldots, m .
$$

It is not difficult to see that for any $z \in \hat{C}$, the number of values of $\mathbf{F}(z)$ cannot be greater than $\sum_{j=1}^{m} k_{j}$. Let $n$ represent the maximum of the value numbers of $F(z)$.

Note that some of the singular points $z_{1}, z_{2}, \ldots, z_{m}$ may be superposed, so the number of different isolated singularities may be less than $m$. Without loss of generality, we may assume that $z_{1}, z_{2}, \ldots, z_{m}$ are distinct points.

Let $T$ be a connected tree on $\hat{C}$, of which the singular points $z_{1}, z_{2}, \ldots, z_{m}$ are just the vertices. It is easy to see that, on the single-connected region $\hat{C} \backslash T$, The multiple-valued function $\mathbf{F}(z)$ is formed with $n$ different single-valued holomorphic branches

$$
F_{1}(z), F_{2}(z), \ldots, F_{n}(z)
$$

and on the boundary of the region $\hat{C} \backslash T, z_{1}, z_{2}, \ldots, z_{m}$ are algebraic singular points of this function. Since $\mathbf{F}(z)$ is a global analytic function, these branches can be obtained by each other through analytical continuation.

For any integer $j=1,2, \ldots, m$ and for any function $g(z)$, which is analytic on $\hat{C} \backslash T$ and has its only singular points at $z_{1}, z_{2}, \ldots, z_{m}$, we may continue this function in the counterclockwise sense along a simple closed path containing only the singular point $z_{j}$ in its interior. When the continuation returns to 
its initial point $z$ the first time, $g(z)$ may be transformed into $\tilde{g}(z)$. Let $M_{j}$ $(j=1,2, \ldots, m)$ represent this transformation, that is,

$$
\tilde{g}(z)=\mathbf{M}_{j} g(z)
$$

Clearly, the operation $\mathbf{M}_{i}$ induces a permutation of the holomorphic system (3.4). The $m$ operations $\mathbf{M}_{i}$, for $i=1,2, \ldots, m$ generate a permutation group, called the monodromy group of the system.

Let

$$
\begin{aligned}
b_{0}(z) & =1, \\
b_{1}(z) & =-F_{1}(z)-F_{2}(z)-\cdots-F_{n}(z), \\
b_{2}(z) & =\sum_{1 \leq i<j \leq n} F_{i}(z) F_{j}(z), \\
& \vdots \\
b_{r}(z) & =(-1)^{r} \sum_{1 \leq i_{1}<i_{2}<\cdots<i_{r} \leq n} F_{i_{1}}(z) F_{i_{2}}(z) \cdots F_{i_{r}}(z), \\
& \vdots \\
b_{n}(z) & =(-1)^{n} F_{1}(z) F_{2}(z) \cdots F_{n}(z) .
\end{aligned}
$$

These functions are in fact the elementary symmetric functions of $F_{1}(z)$, $F_{2}(z), \ldots, F_{n}(z)$. Therefore, under any action of the operations $\mathbf{M}_{1}, \mathbf{M}_{2}, \ldots, \mathbf{M}_{m}$, these symmetric functions are invariant. Notice that, for functions $F_{1}(z)$, $F_{2}(z), \ldots, F_{n}(z)$, points $z_{1}, z_{2}, \ldots, z_{m}$ are the only possible singularities, and they are all algebraic. So $z_{1}, z_{2}, \ldots, z_{m}$ must be the only possible singularities of the symmetric function system (3.6), and they can only be poles. Therefore, these symmetric functions must be rational functions [12]. This means that the $n$-branch functions, $F_{1}(z), F_{2}(z), \ldots, F_{n}(z)$, satisfy the polynomial equation

$$
y^{n}+b_{0}(z) y^{n-1}+b_{2}(z) y^{n-2}+\cdots+b_{n}(z)=0 \text {. }
$$

This proves the theorem.

ACKNOWLEDGMENTS. In preparing this work, the authors have had very useful discussions with Professor He Yuzan, Professor Li Zhong, Professor Zhu Yaochen, and Professor Zhang Xuelian. The referee of this paper has given important comments and substantial detailed suggestions. The authors express heartfelt thanks to all of them.

\section{REFERENCES}

[1] L. V. Ahlfors, Complex Analysis, 3rd ed., International Series in Pure and Applied Mathematics, McGraw-Hill, New York, 1978.

[2] N. G. Čebotarëv, Theory of Algebraic Functions, OGIZ, Moscow, 1948 (Russian). 
[3] H. M. Farkas and I. Kra, Riemann Surfaces, Graduate Texts in Mathematics, vol. 71, Springer-Verlag, New York, 1980.

[4] V. V. Golubev, Lectures on the Analytic Theory of Differential Equations, 2nd ed., Gosudarstv. Izdat. Tehn.-Teor. Lit., Moscow, 1950 (Russian).

[5] E. Goursat, Leçons sur le fonctions hypergeometriques (et sur quelques functions qui s'y rattachent): Intégrales algebriques. Problème d'inversion, Hermann, Paris, 1938 (French).

[6] P. A. Griffiths, Introduction to Algebraic Curves, Translations of Mathematical Monographs, vol. 76, American Mathematical Society, Rhode Island, 1989, translated from the Chinese by K. Weltin.

[7] _ Algebraic Curves, 2nd ed., Peking University Press, China, 2000.

[8] E. Hille, Ordinary Differential Equations in the Complex Domain, Dover Publications, New York, 1997.

[9] E. Kamke, Differentialgleichungen. Lösungsmethoden und Lösungen. I: Gewöhnliche Differentialgleichungen. Neunte Auflage. Mit einem Vorwort von Detlef Kamke, B. G. Teubner, Stuttgart, 1977 (German).

[10] A. I. Markuševič, Theory of Analytic Functions, Gosudarstv. Izdat. Tehn.-Teor. Lit., Moscow, 1950 (Russian).

[11] R. Nevanlinna, Uniformisierung, Die Grundlehren der Mathematischen Wissenschaften in Einzeldarstellungen mit besonderer Berücksichtigung der Anwendungsgebiete, vol. LXIV, Springer-Verlag, Berlin, 1953 (German).

[12] B. P. Palka, An Introduction to Complex Function Theory, Undergraduate Texts in Mathematics, Springer-Verlag, New York, 1991.

[13] L. Schlesinger, Handbuch der Theorie der linearen Differentialgleichungen, Teubner, Leipzig, 1898 (German).

[14] M. F. Singer, Liouvillian first integrals of differential equations, Trans. Amer. Math. Soc. 333 (1992), no. 2, 673-688.

[15] G. N. Watson, A Treatise on the Theory of Bessel Functions, 2nd ed., Cambridge Mathematical Library, Cambridge University Press, Cambridge, 1944.

[16] L. Yinian and Z. Xuelian, Riemann Surfaces, Science Press, Peking, 1997 (Chinese).

Guan Ke-Ying: Department of Mathematics, Northern Jiaotong University, Beijing 100044, China

E-mail address: kyguan@yahoo.com

Lei Jinzhi: Department of Mathematical Science, Tsinghua University, Beijing 100084, China

E-mail address: jzlei@math. tsinghua. edu.cn 


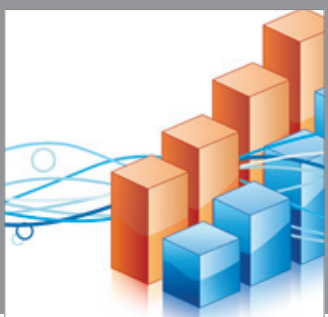

Advances in

Operations Research

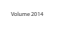

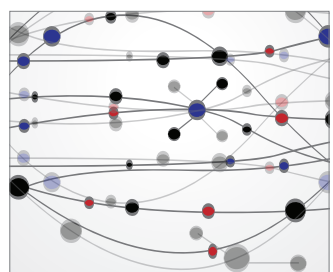

\section{The Scientific} World Journal
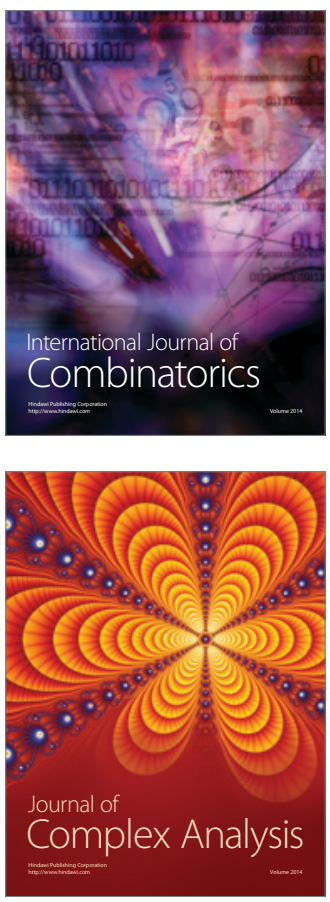

International Journal of

Mathematics and

Mathematical

Sciences
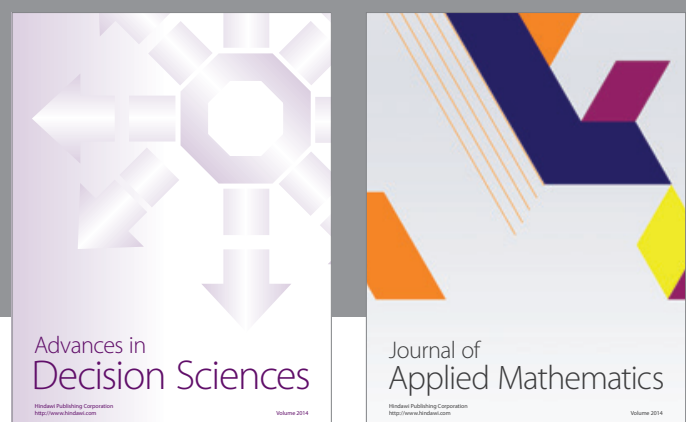

Journal of

Applied Mathematics
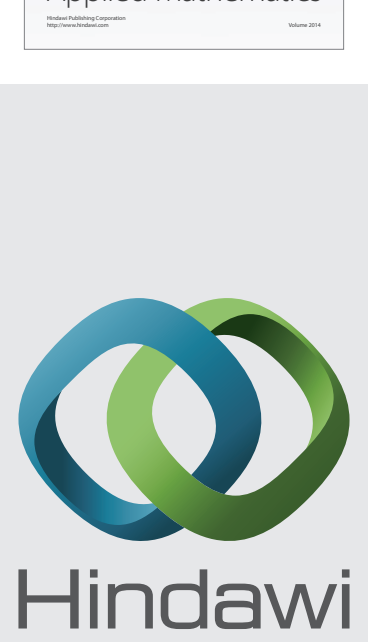

Submit your manuscripts at http://www.hindawi.com
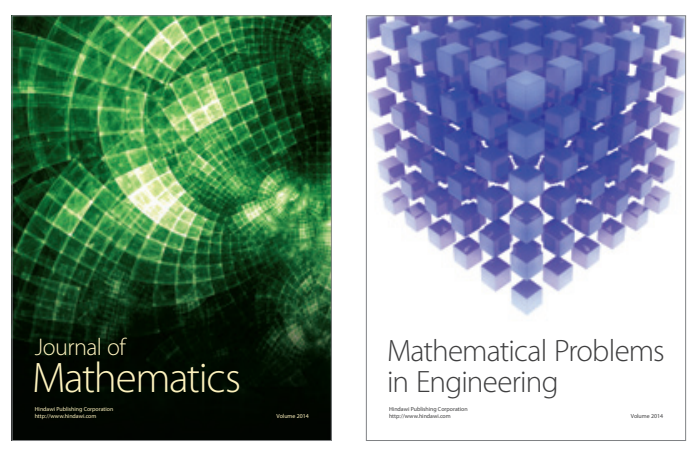

Mathematical Problems in Engineering
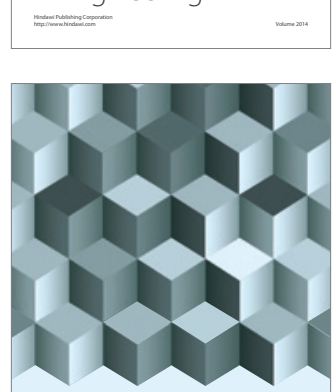

Journal of

Function Spaces
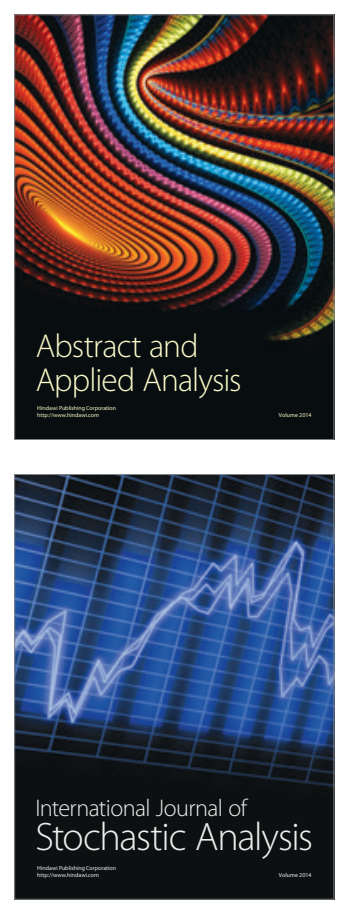

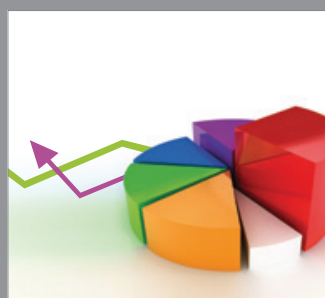

ournal of

Probability and Statistics

Promensencen
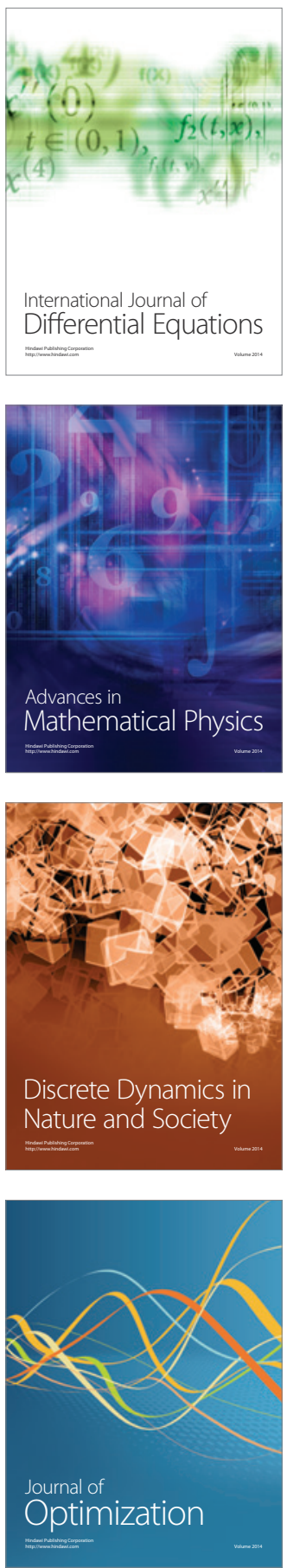